\title{
1 Light enough to travel or wise enough to stay? Brain size evolution and migratory
}

2 behaviour in birds

3 Orsolya Vincze ${ }^{1,2,3}$

4

$5 \quad{ }^{1}$ MTA-DE “Lendület” Behavioural Ecology Research Group, Department of Evolutionary Zoology and

6 Human Biology, University of Debrecen, Debrecen, H-4032, Hungary

$7 \quad{ }^{2}$ Evolutionary Ecology Group, Hungarian Department of Biology and Ecology, Babeş-Bolyai

8 University, Cluj-Napoca, RO-400006, Romania

$9 \quad{ }^{3}$ E-mail: orsolya.vincze@ vocs.unideb.hu

10

11 RUNNING HEAD: Brain size evolution and migration in birds

12 KEY WORDS: Migration, cognition, behavioural flexibility, energy trade-off, innovation

13 DATA ARCHIVING: All data used is included in Supplementary Material S4 


\section{Abstract}

15 Brain size relative to body size is smaller in migratory than in non-migratory birds. Two mutually non-

16 exclusive hypotheses had been proposed to explain this association. On the one hand, the 'energetic

17 trade-off hypothesis' claims that migratory species were selected to have smaller brains because of the

18 interplay between neural tissue volume and migratory flight. In contrast, the 'behavioural flexibility

19 hypothesis' argues that resident species are selected to have higher cognitive capacities, and therefore

20 larger brains, to enable survival in harsh winters, or to deal with environmental seasonality. Here, I test

21 the validity and setting of these two hypotheses using 1,466 globally distributed bird species. First, I

22 show that the negative association between migration distance and relative brain size is very robust

23 across species and phylogeny. Second, I provide strong support for the energetic trade-off hypothesis,

24 by showing the validity of the trade-off among long-distance migratory species alone. Third, using

25 resident and short-distance migratory species, I demonstrate that environmental harshness is associated

26 with enlarged relative brain size, therefore arguably better cognition. My study provides the strongest

27 comparative support to date for both the energetic trade-off and the behavioural flexibility hypotheses,

28 and highlights that both mechanisms contribute to brain size evolution, but on different ends of the

29 migratory spectrum. 


\section{Introduction}

31 Brain size relative to body size has long been considered a major determinant of the cognitive abilities

32 of a given individual, or species (Sol 2009). For instance, large relative brain size has been linked to a

33 wide range of benefits, including increased survival, adaptability to novel environments, innovation

34 propensity, variability of habitats occupied, invasiveness and sociability (Lefebvre et al. 2004, Lefebvre and Sol 2008, Sol 2009, Sol et al. 2007, 2010, Lefebvre 2013). Nevertheless, in spite of the multifaceted benefits conferred by a large brain (relative to body size), there is a downside: high metabolic cost (Sol 2009, Isler and van Schaik 2009). The brain is one of the most energetically expensive organs in the body, consuming up to ten times more energy per unit mass than skeletal muscle (Isler and van Schaik 2006, 2009). Therefore, relative brain size in a given species should reflect a careful balance between costs and benefits; the evolutionary optimum should be the size that maximises survival and reproductive success as a function of species ecology, life history, and behaviour (Sol et al. 2010).

One major ecological constraint on relative brain size across flying homothermic vertebrates is distance travelled during migration (Winkler et al. 2004). This association has repeatedly been demonstrated at the species level in birds (e.g. Winkler et al. 2004, Sol et al. 2005, Vincze et al. 2015), and bats (McGuire and Ratcliffe 2011), as well as at the subspecies level in birds (Cristol et al. 2003, Pravosudov et al. 2007, Fuchs et al. 2015). The correlation is very robust and, in all cases, relative total brain weight decreases with increasing migration distance (Cristol et al. 2003, Winkler et al. 2004, Sol et al. 2005, Pravosudov et al. 2007, McGuire and Ratcliffe 2011, Vincze et al. 2015). The two hypotheses proposed to explain this association are, by definition, explaining variation on two different ends of the migratory distance spectrum (i.e. residents vs. long-distance migrants).The energetic tradeoff hypothesis builds upon the metabolic costs of migration, and that of developing and sustaining neural tissues, suggesting energetic conflict between these two demands (Winkler et al. 2004, McGuire and Ratcliffe 2011). Migration is one of the most energetically challenging periods in a bird's life: Bartailed Godwits (Limosa lapponica), for example, cover 11,000 km in a single non-stop flight (Gill et al. 2009). Such strenuous movements are often on the edge of avian physiological endurance and necessitate a range of adaptations to make the journey possible (Hedenström 2010). For instance, we know that body mass is often doubled during the pre-migratory fattening process to support the 
60 energetic needs of the journey (Newton 2008), while almost all organs undergo significant size

61 reduction prior to migration to minimise the metabolic cost of transport (Piersma and Lindström 1997,

62 Battley et al. 2000). An extensive study on the morphological adaptations to migration in birds found

63 that heart size, the most calorie-hungry structure in the body, is relatively smaller in long-distance

64 migrants (Vágási et al. 2016), corroborating negative selection on energetically expensive organ sizes.

65 Given the energetic cost of flight, long-distance migration may compromise a bird's ability to support

66 the high metabolic cost of a large brain. Thus, the energetic trade-off hypothesis predicts directional

67 selection that favours smaller relative brain size with increasing migration distance.

69 In contrast, the behavioural flexibility hypothesis assumes a positive directional selection on relative

70 brain size in resident birds (Winkler et al. 2004) instead of negative selection in migrants. Resident bird

71 species often experience strong spatial and temporal fluctuations in their environments, and therefore

72 tend to rely more heavily on novel food sources, exploited through innovations and learning (Sol et al.

73 2005, Aplin et al. 2013). Classical examples of such behaviours are the 'milk bottle' innovation in Blue

74 Tits (Cyanistes caeruleus) (Aplin et al. 2013), or Great Tits (Parus major) predating on hibernating bats

75 (Estók et al. 2009). Such behaviours usually only happen under environmental conditions (e.g. harsh

76 winters) that limit normal food sources (Estók et al. 2009); this also highlights the importance of

77 innovation in seasonal and harsh environments. Indeed, innovation propensity and associated relative

78 brain size (Timmermans et al. 2000, Reader and Laland 2002), are both highest in resident species and

79 lowest in long distance migrants (Sol et al. 2005). Nonetheless, whether higher degrees of innovation in

80 resident species reflects necessity, or their capacity, has yet to be determined. To attempt to address this,

81 a reformulation of the behavioural flexibility hypothesis by inverting causalities was coined the

82 'migratory precursor hypothesis' (Sol et al. 2005). The elevated cognitive capacity of large-brained

83 birds would enable them to be residents, while small-brained species are forced to migrate. Irrespective

84 of causality, higher cognitive needs, especially if innovative behaviour is socially transmitted (Aplin et

85 al. 2013), may represent one plausible explanation for the larger relative brain sizes of resident bird

86 species and might represent a coping mechanism to harsh or seasonal environments.

87

88 Exploring the relationship between relative brain size and the environmental harshness, or variability, 89 experienced by resident birds in different climatic zones, or latitudes, could provide a strong test of the 
90 behavioural flexibility/migratory precursor hypothesis (Winkler et al. 2004). Studies investigating the

91 behavioural flexibility hypothesis to date are, however, scant and results are contradictory (Schuck-

92 Paim 2008). In neotropical parrots, climate variability was shown to be positively associated with

93 relative brain size (Schuck-Paim 2008), which provides some intraspecific support for the behavioral

94 flexibility hypothesis. Moreover, elevated winter harshness and the associated increased requirement

95 for food-caching is correlated with enlargement of brain regions responsible for spatial memory in

96 different Black-capped Chickadee (Poecile atricapillus) populations (Roth and Pravosudov 2009, Roth

97 et al. 2011). There is thus some evidence that the environmental harshness and fluctuation influence

98 brain evolution and functionality; however, which aspects of the environment are most important in this

99 respect, and in what settings do selective forces act, remain unanswered. By extending the geographical

100 and taxonomic coverage of previous studies, and by testing how different environments experienced by

101 species with similar migratory behaviours result in relative brain size differentiation could provide

102 potential answers to these questions.

103

104 In this study, I test separately the validity of the energetic trade-off and behavioural flexibility

105 hypotheses, and explore the nature of the negative correlation between relative brain size and migratory

106 behaviour in birds. First using data from the literature, I assess the generality of this negative

107 association on the basis of an extensive list of bird species $(n=1,466)$, across a very wide body size

$108(2.7 \mathrm{~g}-44 \mathrm{~kg})$ and taxonomic range, encompassing ratites to passerines. Second, using migration

109 measured on a continuous scale $(0 \mathrm{~km}-13,063 \mathrm{~km})$ and species with migration distance $>0 \mathrm{~km}$ (i.e.

110 excluding residents), I test the validity of the energetic trade-off hypothesis. This hypothesis will gain

111 support if there is a negative association between migration distance and relative brain size, and the

112 effect will be strongest among long-distance migratory birds. Third, using only resident species $(\mathrm{n}=$

113 937), across a tropical-to-arctic distributional spectrum, I test the validity of the behavioural flexibility

114 hypothesis. In this final case, I use winter minimum temperature, seasonality of ambient temperature,

115 and wintering latitude to test which one of these factors best predicts the relative brain size of resident

116 birds. The behavioural flexibility hypothesis will be supported in cases where relative brain size

117 increases with latitude or seasonality, or decreases with increasing winter minimum temperature; note

118 that the latter ought to have the strongest effect when the nature of this association is defined by

119 environmental harshness. My study thus provides the first broad and fine scale mutual test for the 
120 behavioural flexibility and energy trade-off hypotheses, to explore how these mechanisms shape the 121 evolution of avian brains.

123 Materials and Methods

124 BRAIN AND BODY SIZE

125 I extracted brain and body weight data from Iwaniuk and Nelson (2003), a primary dataset listing the

126 endocranial volumes of a wide range of birds. Endocranial volume is a highly reliably method to

127 measure brain size both across, and within, species (Iwaniuk and Nelson 2002). Brain mass was then

128 obtained by multiplying reported endocranial volumes by the density of fresh brain tissue, $1.036 \mathrm{~g} / \mathrm{mL}$

129 (Iwaniuk and Nelson 2003), and the dataset was then further modified by calculating a single mean for

130 species for which data was originally reported at the subspecies level (e.g. Platycercus elegans elegans

131 and P. e. flaveoulus), or using two synonymous names (e.g. Esacus magnirostris and E. neglectus). In

132 these cases, species means were calculated as the weighted arithmetic mean of separate measurements

133 where weights were represented by the number of specimens measured in each case.

135 Given that larger-bodied species have larger brains, body mass needs to be controlled for when 136 comparing brian sizes across species (Lashley 1949). Body mass was therefore included in each of the 137 models presented here, as brain size relative to body size is a measure that reflects the surplus of neural 138 tissue versus the amount required for basic bodily functions (Lashley 1949), and is associated with a 139 range of cognitive traits across species (see above).

\section{MIGRATION DISTANCE}

142 Distribution maps (shape files) for each species were downloaded from

143 http://www.birdlife.org/datazone/info/spcdownload (BirdLife International and NatureServe 2014), and

144 the geometric centroid of the spatial polygon of breeding (breeding and resident) and wintering

145 (wintering and resident) ranges were calculated using the ' $\mathrm{gCentroid}$ ' function in the R package 'rgeos'

146 (Bivan and Rundel 2013). Migration distance was calculated as the geographic distance between

147 breeding and wintering centroids using a custom function written in R (Vágási et al. 2016). 'Migratory

148 distance' thus denotes the average distance travelled by a given species during migration. Additionally,

149 I extracted the latitude of the non-breeding centroids, and calculated absolute values (thereafter, 'non- 
breeding latitude').

\section{ENVIRONMENTAL HARSHNESS AND SEASONALITY}

153 I extracted ambient temperature data from the University of East Anglia Climate Research Unit 154 database (CRU, http://www.cru.uea.ac.uk/; version 3.10.01; Mitchell and Jones 2005), a global dataset containing interpolated monthly average land temperatures $\left({ }^{\circ} \mathrm{C}\right)$ from 1901 onwards in a grid of spatial coordinates $(0.5 \times 0.5$ degrees $)$. I used the most recent temperature data ('cru_ts_3.23.2011.2014', downloaded on 26 September 2015) comprising monthly temperature means from 2011 to 2014. First, I averaged these four years to calculate mean monthly temperatures for each spatial grid cell. From the resulting data file, I created a 12-layer shape file, where each layer contained a month's mean temperature separately for each cell. Third, by intersecting temperature and species distribution shape files, I calculated the monthly mean temperatures for each species, separately for their wintering and breeding grounds. This resulted in 12 monthly means on the breeding ground, and 12 monthly means on the wintering ground for each species. For the wintering ground, I extracted the lowest monthly mean (thereafter, 'non-breeding minimum temperature') as a proxy of winter harshness. 'Seasonality' was calculated as the difference between the lowest and highest monthly mean temperatures on the breeding ground, and thus it reflects the extent of maximum thermal fluctuation during the course of a year on the breeding ground for each species. Note that neither non-breeding minimum temperature nor seasonality reflects the true environmental conditions experienced by species with migration distances greater than $0 \mathrm{~km}$. This is so, because long-distance migrants often do not experience the coldest periods on the wintering grounds (e.g. July in South-America for White-rumped Sandpiper Calidris fuscicollis), nor do they on the breeding grounds (e.g. January in North-America for the same species).

172 The latter is true for short-distance migrants too, due to their migratory tendencies under harsh 173 environmental conditions., Therefore, both non-breeding minimum temperature and seasonality were 174 only used in models based on species that have a migration distance of less than $1,000 \mathrm{~km}$; the true 175 values of non-breeding minimum temperature and seasonality experienced by long distance migrants 176 could not be calculated due to lack of information on their temporal migratory patterns. In addition, 177 because non-breeding minimum temperature and seasonality could not be calculated for three fully 178 resident species with extremely restricted distributions (i.e., Anas laysanensis, Porzana atra, Vini 179 stepheni), sample size slightly varies between models with different explanatory variables. 


\section{PHYLOGENY}

182 In order to implement the similarity of species due to common descent, I controlled for phylogenetic

183 relatedness in all analyses. To do this I downloaded 100 random trees from www.birdtree.org (Jetz et al.

184 2012) using the Hackett backbone tree (Hackett et al. 2008), and repeated every model with each of

185 these random trees to control for phylogenetic uncertainty (Rubolini et al. 2015).

187 STATISTICAL ANALYSES

188 I performed phylogenetic generalised least squares regressions (PGLS) using the 'pgls' function as 189 implemented in the R package 'caper' (Orme et al. 2013). Brain size was used as a dependent variable 190 in double predictor models, containing body mass and one of the following covariates: migration 191 distance, non-breeding minimum temperature, seasonality or non-breeding latitude. To test for the 192 energetic trade-off hypothesis, I built double predictor models containing migration distance and body 193 mass as explanatory variables on subsets of species over seven different migration distance intervals 194 (i.e. migration distance $\geqslant 0 \mathrm{~km},>0 \mathrm{~km},>500 \mathrm{~km},>1,000 \mathrm{~km},>2,000 \mathrm{~km},>0 \mathrm{~km} \&<500 \mathrm{~km},>500 \mathrm{~km}$

$195 \&<1,000 \mathrm{~km})$. However, because the effect of migration distance might be confounded by the effect of 196 climate, given that these two often covary (i.e. species with longer migration distances experience 197 milder wintering climates), I repeated the above model using a subset of species with migration 198 distance $>2,000 \mathrm{~km}$, and with non-breeding range centroids within the tropics ( $\left.23.4^{\circ} \mathrm{N}-23.4^{\circ} \mathrm{S}\right)$. I

199 expect the strongest effect of migration distance on brain size in species with the longest migratory 200 trajectories if the energetic trade-off hypothesis is to be supported. Further, to test the behavioural 201 flexibility hypothesis, I built double predictor models containing body mass and non-breeding 202 minimum temperature, seasonality or non-breeding latitude, as explanatory variables on subsets of 203 species with five different migration distance intervals (i.e. migration distance $=0 \mathrm{~km},<500 \mathrm{~km}$, $204<1,000 \mathrm{~km},>0 \mathrm{~km} \&<500 \mathrm{~km},>500 \mathrm{~km} \&<1,000 \mathrm{~km}$ ). I expect the strongest effect of all three 205 variables in fully resident species, and that the strength of these associations will decrease with the 206 length of migration distance.

208 All the above analyses were repeated using passerines only, since these perching songbirds (order 209 Passeriformes) are less variable morphologically and trace their origins to a more recent common 
210 ancestor than the non-passerines, but are more speciose and exhibit an impressive array of cognitive

211 abilities and migratory strategies (Sol et al. 2005). Moreover, models were repeated using non-

212 passerine bird orders with sufficient number of species and with considerable variance in the focal

213 explanatory variable. These orders were the Anseriformes and Charadriiformes for the energetic trade-

214 off hypothesis and Piciformes, Strigiformes and Galliformes for the behavioural flexibilty hypothesis.

215 Taxonomic order was obtained using the 'tax_name' function as implemented in the R package 'taxize'

216 (Chamberlain et al. 2014), and each of the models described above was repeated with 100 random

217 phylogenetic trees; AICc scores extracted and AICc weights were calculated. AICc weights were then

218 used to calculate weighted mean $\mathrm{t}$ and $\mathrm{p}$-values across the 100 models; distributions of both $\mathrm{t}$ and $\mathrm{p}$

219 values of the focal explanatory variables in these model sets were plotted and are reported in

220 Supporting Information S1 (Table 1), and Supporting Information S2a and S2b (Table 2) for the entire

221 species and passerines respectively. Phylogenetic dependence was estimated using Pagel's $\lambda$, set to the

222 most appropriate value assessed by maximum likelihood in each model. Brain mass and body mass

223 were log-transformed prior to analyses, all other variables were used untransformed.

225 Migratory species often accumulate large amounts of fat to support their migratory flights (Newton

226 2008). Such body mass fluctuations might bias the results of brain size analyses in cases where

227 migratory species have larger body masses recorded in the dataset due to accumulated fuel reserves. In

228 these cases, relative brain size in longer distance migrants (with more fuel accumulated) would be

229 estimated erroneously as smaller. To rule out this confounding effect, I first tested whether mean body

230 mass used in the brain size models is correlated with migration distance $(n=1,466)$. Second, I obtained

231 data on minimum and maximum body mass from Dunning (2008) for 1,131 bird species present in the

232 brain dataset. Minimal and maximal body masses were obtained by averaging sexes, subspecies, and

233 populations if separate values were available, and on the basis of these data I tested whether the ratio

234 between minimum and mean body mass, as well as the ratio between maximum and mean body mass

235 covaries with migration distance. All three of these models were tested in a PGLS framework, using

236100 phylogenetic trees. Results reported are weighted means (by AICc weights) of t- and P-values

237 calculated across the 100 models. Model averaging was performed in the same fashion as with brain

238 size models, and mean body mass and the two body mass ratios were all log-transformed prior the

239 analyses. 
241 Graphical presentation of data was done using residual brain masses, calculated form a log-log standard

242 linear regression between brain mass and body mass. Fitted lines and associated standard errors were

243 obtained from the PGLS model between the residual brain mass and the focal predictor variable, and

244 standard errors were obtained using the 'predictSE.gls' function as implemented in R package

245 'AICcmmodavg' (Mazerolle 2015). P values were not adjusted for multiple comparison, in order to

246 avoid inflation of the type II error probability (Rothman 1990, 2014). All statistical analyses and

247 graphical representations of results were carried out in R 3.2.0 (R Core Team 2015) and all data used in

248 the analyses are reported in Supporting Information S4.

250 Results

\section{DATA-SET COVERAGE}

252 Across the dataset (Fig. 1), migration distance varied from $0 \mathrm{~km}(\mathrm{n}=937$ species) to 13,063 $\mathrm{km}$ in the

253 White-rumped Sandpiper (Calidris fuscicollis), while non-breeding latitude varied from $74.61^{\circ}$ in the

254 Ivory Gull (Pagophila eburnea) to $0.01^{\circ}$ in the Spot-winged Antbird (Schistocichla leucostigma). In

255 species with migration distance $<1,000 \mathrm{~km}$, non-breeding minimum temperature ranged from $-29.05^{\circ} \mathrm{C}$

256 in the Rock Ptarmigan (Lagopus muta) to $26.84^{\circ} \mathrm{C}$ in the Northern Screamer (Chauna chavaria), while

257 seasonality varied from $0.42^{\circ} \mathrm{C}$ in the Eyebrowed Jungle-flycatcher (Rhinomyias gularis) to $44.75^{\circ} \mathrm{C}$ in

258 the Asian Rosy Finch (Leucosticte arctoa).

\section{THE ENERGY TRADE-OFF HYPOTHESIS}

261 Brain size is strongly negatively correlated with migration distance across the entire migratory

262 spectrum (Table 1, Fig. 2A,D). This association disappears when fully resident species (migration

263 distance $=0 \mathrm{~km}$ ) were excluded from analyses (Table 1). Indeed, when analyses were restricted to short

264 distance migrants $(0-1,000 \mathrm{~km})$, the negative association between brain size and migration distance did 265 not emerge (Table 1, Fig. 2B,E) indicating that short distance migrants do not fit the relative brain size

266 - migration distance continuum when this is assessed using the entire migratory spectrum.

267 Nevertheless, the strength of the negative association between brain size and migratory distance 268 increased again, despite a reduction in sample size, after short-distance migrants were excluded (i.e.

269 subsets with migration distance from $>500 \mathrm{~km}$ to $>2,000 \mathrm{~km}$, Table 1 and Fig. 2C,F). The negative 
270 association between migration distance and brain size was also strong for the subset of species with

271 tropical wintering centroids and migration distances over 2,000 km. All results were highly consistent

272 when repeated just for passerines (Table 1, Fig. 2). Similar pattern was found in the case of

273 Charadriiformes and Anseriformes, but these associations did not reach significance (Supporting

274 Information S3, Table S1, Figure S1).

275

276 Mean body mass is negatively associated with migration distance (PGLS, $\mathrm{n}=1,466, \mathrm{t}=-2.25, \mathrm{p}=$ 277 0.0035), indicating that longer distance migratory species have lower, not higher, average body masses

278 than species with shorter migratory distances. The ratio between minimum and mean body mass

279 decreased slightly with migration distance (PGLS, $\mathrm{n}=1,131, \mathrm{t}=-2.54, \mathrm{p}=0.0120$ ), while the ratio

280 between maximum and mean body mass was strongly positively associated with migration distance

$281 \quad($ PGLS, $\mathrm{n}=1,131, \mathrm{t}=3.42, \mathrm{p}=0.0008)$.

282

\section{THE BEHAVIOURAL FLEXIBILITY HYPOTHESIS}

285 Non-breeding minimum temperature has a strong effect on brain size in both fully resident and short-

286 distance migratory species (Table 2, Fig. 3); the lower the non-breeding minimum temperature, the

287 larger the brain size (Table 2 and Fig. 3). Indeed, the effect of non-breeding minimum temperature was

288 comparable across different migratory intervals between 0 and $500 \mathrm{~km}$, but not above $500 \mathrm{~km}$ (Table

289 2). In several species subsets, non-breeding minimum temperature is the only significant predictor of

290 relative brain size, while seasonality and non-breeding latitude have little predictive power. Where

291 significant, brain size increases with seasonality and increases with increasing non-breeding latitude

292 (Table 2); all results were highly consistent when repeated using just passerines (Table 2). Moreover,

293 results were highly consistent for the Piciformes and the Strigiformes bird orders, but none of the tested

294 environmental variables influenced brain size in the Galliformes bird order (Supporting Information

295 S3, Table S2, Figure S2).

\section{Discussion}

298 In this study, I show in the first place that whole brain size in birds is negatively correlated with

299 migration distance. This key result corroborates earlier studies (Sol et al. 2005, 2010, McGuire and 
300 Ratcliffe 2011), but extends this negative correlation across much wider taxonomic and geographic

301 scales, and provides a basis for the generalisation of this association outside passerines. Secondly, my

302 study provides strong and clear support for the validity, and context, of the two alternative hypotheses

303 explaining the association between brain size and migration distance in birds, the energetic trade-off

304 and the behavioural flexibility hypotheses.

305

\section{ENERGETIC TRADE-OFF HYPOTHESIS}

307 Relative brain size in birds strongly decreases with increasing migration distance; this is true when 308 considering the entire migratory spectrum, or just long-distance migrants. First, results based on the 309 entire migratory spectrum corroborate earlier studies (Sol et al. 2005, 2010, McGuire and Ratcliffe 310 2011), and provide a basis for generalising the negative association between relative brain size and 311 migration distance across all birds. Note however, that the negative association between brain size and 312 migration distance was not significant in the two non-passerine bird orders tested, however both of

313 these shower similar patterns. Second, results based just on long-distance migrants provide the 314 strongest support yet for the energetic trade-off hypothesis, indicating that it exists not just among 315 major migration distance subdivisions (e.g. residents, short-, and long-distance migrants), but also on a 316 fine scale within just long-distance migrants. One major drawback of earlier studies is that they 317 categorised species based on the length of their migratory trajectories; long-distance migrants were 318 handled within just one (Sol et al. 2005, McGuire and Ratcliffe 2011), or few categories (Sol et al. 319 2010). Here, I provide support for the energetic trade-off hypothesis by exploring fine-scale variations 320 of both migration distance and relative brain size within these categories, and results show that short321 distance migrants do not fit on the relative brain size - migration distance linear continuum. This 322 suggests that two different mechanisms control the evolution of the disparate relative brain sizes found 323 in migrants and residents, and that these mechanisms act on separate ends of the migratory spectrum. In 324 resident birds it is cognitive needs, while in migrants, energetic limitations appear to be important in 325 regulating brain size evolution. Results suggest that shorter-distance migrants are only partially affected 326 by both of these mechanisms.

328 Due to the correlative nature of this study, the negative association between migration distance and 329 relative brain size could potentially be confounded by several factors. First, the longer the migration 
330 distance, the milder wintering conditions can get; therefore, the smaller brain size in long-distance

331 migrants could also be explained by the year-round milder environment these birds experience. Note

332 however that the negative association between brain size and migration distance is also apparent in

333 species with migration distances over 2,000 km, and with wintering range geometric centroids within

334 the tropics. This result suggests that the negative association between brain size and migration distance

335 is unlikely to be confounded by correlated climate effects, and that the nature of this association is

336 indeed an energetic trade-off. Second, given that migratory species often accumulate large fat reserves

337 to support their migratory flight, relative brain size might be underestimated if lean body mass is

338 overestimated in migrants. Such errors in the data could lead to a false identification of the energetic

339 trade-off hypothesis as true, given that larger fuel amounts are accumulated in longer distance migrants.

340 Note however, that mean body mass used in the analyses was actually negatively correlated with

341 migration distance in this dataset, indicating the migratory fuelling did not affect mean body mass

342 estimates used here. Additionally, for a subsample of species $(n=1,131)$ the ratio of minimum to mean

343 body mass decreased slightly with increasing migration distance. This weak association, compared to

344 the strong positive association between migration distance and the ratio of maximum to mean body

345 mass indicates that migratory fuelling is unlikely to largely distort mean body mass values used here

346 and is therefore unlikely to confound my results.

348 An earlier phylogenetic path analyses showed that the largest fraction (68\%) of the correlation between

349 relative brain mass and migratory distance is a direct effect of migration on brain size (Sol et al. 2010).

350 Although these authors argued that brain size reduction in migrants could have originated from the

351 lowered importance of cognitive capacities in these birds (Sol et al. 2010), relative brain size in short-

352 distance migrants is not affected by migration distance. This result is important because cognitive needs

353 for resource exploitation in short-distance migrants might arguably be closer to those of long-distance

354 migrants than to those of residents simply because of their migratory tendencies in case of resource

355 shortages and their potentially decreased needs for innovation (e.g. irruptive or facultative migration,

356 Newton 2008). Therefore, it is unlikely that the brain size of long-distance migrants shrinks simply

357 because of a reduction in cognitive need, leaving the energetic trade-off hypothesis as a more plausible

358 explanation. This is especially the case given that the negative association between migration distance

359 in long-distance migratory species (over2,000 km) and with tropical non-breeding ranges still holds 
360 true, although cognitive needs within this group of birds could potentially be similar. Second, given that 361 migration is an extremely strenuous activity (Hedenström 2010), and the length of migration distance

362 was shown to negatively correlate with the energetically expensive heart size (Vágási et al. 2016), I

363 consider the pure energetic trade-off hypothesis to be the most likely explanation of brain size

364 reduction in long-distance migrants.

\section{THE BEHAVIOURAL FLEXIBILITY HYPOTHESIS}

367 Non-breeding minimum temperature is a strong predictor of relative brain size in fully resident $(\mathrm{n}=$ 368934 species), and short-distance (up to $500 \mathrm{~km}, \mathrm{n}=142$ species) migratory birds. In other words, the 369 colder the minimum monthly temperature on the wintering ground the larger the relative brain size of 370 birds. Additionally, relative brain size significantly increased with non-breeding latitude and 371 seasonality although these effects are weaker than the association with non-breeding minimum 372 temperature. Thus, my results strongly indicate that winter harshness is associated with larger brains 373 across the avian phylogeny. Given that non-breeding latitude and seasonality have weaker effects than 374 non-breeding minimum temperature on relative brain size, it is more likely that environmental severity 375 reflected by low ambient temperature, high snow cover, and/or reduced day length (Roth and 376 Pravosudov 2009), rather than the seasonal nature of the environment being the strongest selective 377 force on brain size evolution in resident birds. Indeed, the importance of climate severity in brain 378 evolution has previously been reported; food- caching Black-capped Chickadees from harsher 379 wintering ranges have better spatial memory, as well as larger hippocampi and higher neuronal density 380 in these brain regions responsible for this skill (Roth and Pravosudov 2009, Pravosudov and Clayton 381 2002, Roth et al. 2011). Enhanced spatial memory is thus a potential mechanism enabling birds to cope 382 with environmental harshness, especially in food-caching species. The hippocampus occupies just a 383 small part of total brain volume, however, and thus the results presented here must reflect additional 384 neural adaptations to environmental severity. To date we have very limited knowledge on how 385 environmental conditions, in particular which aspects of the environment and in which way does it 386 influence brain and cognitive evolution across species. The topic therefore deserves considerable future 387 scientific attention. 
390 Change in the size of different brain regions with migration distance is non-uniform (McGuire and

391 Ratcliffe 2011, Fuchs et al. 2014, Vincze et al. 2015), and reduction of whole brain size with increasing

392 migration distance is mostly accounted for by reduction in the size of the telencephalon (Vincze et al.

393 2015), the center of higher cognitive processes. In line with the latter results, Fuchs et al. (2015) found

394 that migratory lark sparrows (Chondestes grammacus) showed a clear trend toward having larger

395 nidopalliums (a central neural substrate of higher cognitive processes in birds) than residents of the

396 same species. Therefore, it is probable that the larger relative brain size of resident birds compared to

397 migrants is indeed associated with their larger telencephalon and better cognitive abilities that could

398 enhance their survival probability especially under harsh environmental conditions. It would be

399 insightful then to consider how environmental harshness in various resident birds influences the

400 evolution of different brain regions, on a cross-species scale. Such a follow-up study could provide

401 more precise insights into whether increase in the size of telencephalon (and regions thereof, e.g.

402 hippocampus) is specifically selected in species wintering under harsher environmental conditions.

403 Additionally, whether brain size enlargement preceded, or followed, the switch in migratory habit in

404 avian evolution is yet to be determined. Pravosudov et al. (2007) for instance examined three

405 subspecies of white-crowned sparrow (Zonotrichia leucophrys) and showed that it is more likely that

406 brain size enlargement took place after the switch from migratory to sedentary behaviour. Nonetheless,

407 further studies should examine the nature if these associations on a broader taxonomic scale.

408

409 Relative brain size variation is subtler in bats than in birds (McGuire and Ratcliffe 2011), and the

410 authors suggest that this discrepancy could originate from the shorter migration distances covered by

411 bats relative to birds. In addition, I suggest that besides the longer migration distances selecting for

412 smaller brains in both birds and mammals, harsh environments experienced by vigilant resident birds

413 (but not hibernating resident bats) will select for enlarged brains, further distancing relative brain

414 volume of resident from that of long-distance migratory birds.

415

416 Here I show that both environmental harshness and migration distance strongly affect brain size

417 evolution in birds. It's important to note however that these two factors appear to explain only a

418 fraction of the cross-species variance observed (see Fig 2,3). The extra variation is certainly explained

419 by other social, ecological, physiological or life-history factors not examined here that affect brain or 
cognitive evolution across birds.

422 Importantly, comparative studies of full brain size have been subject to strong criticism in recent years 423 (Healy and Rowe 2007). The argument is that the brain is responsible for a wide range of functions, 424 therefore is not suitable to directly associate it with specific behaviours. However, a range of studies 425 indicate that relative brian size is a strong predictor of cognitive abilities, such as innovativity, learning, 426 invasion, tool use, memory, variability of habitats occupied (Schuck-Paim 2008, Sol 2009, Sol et al. 427 2007, 2010). Moreover, recent comparative evidence reveals that large brains in birds are a result of 428 disproportionately enlarged pallial areas known to play key roles in avian cognition (Sayol et al. 2016). 429 These studies suggest that whole brain size is indeed a useful tool of assessing general evolutionary 430 patterns of brain and cognitive evolution. The results obtained this way will naturally benefit from a 431 more specific research framework, where the change in specific brain regions is precisely assessed. 432

\section{CONCLUSIONS}

434 Here I demonstrate that increasing environmental harshness during the non-breeding period is 435 associated with larger relative brain sizes in both resident and short distance migrants and thus, in these 436 species, selection for behavioural flexibility must be an important driver of brain size evolution.

437 Nevertheless, because I also show that increasing migration distance is linked with decreased relative 438 brain size, the energetic trade-off hypothesis is also supported, especially in species with long 439 migratory flights. Taken together, this study illustrates that the selection for larger brain size by cold 440 wintering temperatures and the selection for smaller brain size by migratory flight both contribute to 441 the evolution of disparate relative brain sizes of migratory and resident bird species, and these two 442 mechanisms act on different ends of the migratory spectrum. Finally, it is important to note that I have 443 taken a correlative approach here, therefore the nature of causalities cannot be inferred from my results. 444 In other words, migratory habit or geographic distributions may select for larger or smaller brains, but 445 brain size evolution might as well precede switch in migratory strategy or define suitable distribution 446 ranges (Sol et al. 2005, Pravosudov et al. 2007).

\section{ACKNOWLEDGEMENTS}

449 I thank CI Vágási and PL Pap for motivating me to write up this study and for constructive discussions. 
450 CI Vágási, and Z Németh made helpful comments on earlier versions of the manuscript. I'm very 451 grateful to GJ Dyke who kindly corrected and polished my English. I thank Associate Editor Matthew 452 Carling and the four anonymous reviewers for their constructive criticism, which helped me to 453 significantly improve this manuscript. The author was supported by the Hungarian Ministry of Human 454 Resources, National Talent Program (NTP-EFÖ-P-15-0043), by the 455 Hungarian Eötvös Scholarship (MÁEÖ2016_15/76740) and by the Hungarian Research Fund OTKA 456 \#K113108 to ÁZ Lendvai. I declare no conflict of interests.

\section{SUPPORTING INFORMATION}

459 Supporting Information S1: These figures represent parameter distribution over 100 models with 460 different phylogenetic trees. Each row of the figure represents a row from Table 1. The first two 461 columns represent t- and P-values from models of the entire species pool, while the other two from 462 models of Passerines.

463 Supporting Information S2: These figures represent parameter distribution over 100 PGLS models 464 with different phylogenetic trees. Each row of the figure S2a represents a row from the first part of 465 Table 2 (i.e. entire species pool), while S2b from the second part of Table 2 (i.e. Passerines). $1^{\text {St }}$ and $2^{\text {nd }}$ 466 columns represent parameters from models containing non-breeding minimal temperature, $3^{\text {rd }}$ and $4^{\text {th }}$ 467 columns from models containing seasonality, $5^{\text {th }}$ and $6^{\text {th }}$ columns from models containing non-breeding 468 latitude.

469 Supporting Information S3: Models exploring the relationship between brain size and migration 470 distance (in two non-passerine bird-orders) as well as with non-breeding minimal temperature, 471 seasonality and non-breeding latitude (in three non-passerine bird orders). Results of models and 472 graphical presentation are both given.

473 Supporting Information S4: Data used in the analyses.

\section{LITERATURE CITED}

476 Aplin, L. M., B. C. Sheldon and J. Morand-Ferron. 2013. Milk bottles revisited: social learning and individual variation in the blue tit, Cyanistes caeruleus. Anim. Behav. 85:1225-1232.

478 Battley, P. F., T. Piersma, M. W. Dietz, S. Tang, A. Dekinga and K. Hulsman. 2000. Empirical evidence for differential organ reductions during trans-oceanic bird flight. Proc. R. Soc. Lond. B 
267:191-195.

481 Bartoń, K. 2015. MuMIn: Multi-Model Inference. R package version 1.15.1. http://CRAN.Rproject.org/package=MuMIn

483 BirdLife International and NatureServe. 2014. Bird species distribution maps of the world. BirdLife International, Cambridge, U.K.

485 Bivand, R. and C. Rundel. 2013. Rgeos: Interface to Geometry Engine - Open Source (GEOS). R Package Version 0.3-2.

487 Chamberlain, S., E. Szocs, C. Boettiger, K. Ram, I. Bartomeus and J. Baumgartner. 2014. taxize: Taxonomic information from around the web. $\mathrm{R}$ package version 0.3.0. https://github.com/ropensci/taxize

Cristol, D. A., E. B. Reynolds, J. E. Leclerc, A. H. Donner, C. S. Farabaugh and C. W. S. Ziegenfus. 2003. Migratory dark-eyed juncos, Junco hyemalis, have better spatial memory and denser hippocampal neurons than non-migratory conspecifics. Anim. Behav. 66:317-328.

Estók, P., S. Zsebők and B. M. Siemers. 2009. Great tits search for, capture, kill and eat hibernating bats. Biol. Lett. 6:59-62.

Fuchs, R., H. Winkler, V. P. Bingman, J. D. Ross and G. Bernroider. 2014. Brain geometry and its relation to migratory behavior in birds. J. Adv. Neurosci. Res 1:1-9.

Fuchs, R., V. P. Bingman, J. D. Ross and G. Bernroider. 2015. Brain contrasts between migratory and nonmigratory North American lark sparrows (Chondestes grammacus). NeuroReport 26:10111016.

Gill, R. E. Jr., T. L. Tibbitts, D. C. Douglas, C. M. Handel, D. M. Mulcahy, J. C. Gottschalck, N.Warnock, N. J. McCaffery, P. F. Battley and T. Piersma. 2009. Extreme endurance flights by landbirds crossing the Pacific Ocean: ecological corridor rather than barrier? Proc. R. Soc. Lond. B 276:447-457.

Hackett, S. J., R. T. Kimball, S. Reddy, R. C. K. Bowie, E. L. Braun, M. J. Braun, J. L. Chojnowski, W. A. Cox, K. L. Han, J. Harshman et al. 2008. A phylogenomic study of birds reveals their evolutionary history. Science 320:1763-1768.

509 Hedenström, A. 2010. Extreme endurance migration: what is the limit to non-stop flight. PLoS Biol, 
511 Isler, K. and C. van Schaik. 2006. Costs of encephalization: the energy trade-off hypothesis tested on birds. J. Hum. Evol. 51:228-243.

513 Isler, K. and C. P. van Schaik. 2009. The expensive brain: a framework for explaining evolutionary $514 \quad$ changes in brain size. J. Hum. Evol., 57:392-400.

515 Iwaniuk, A. N., and J. E. Nelson. 2002. Can endocranial volume be used as an estimate of brain size in birds? Can. J. Zoolog. 80:16-23.

517 Iwaniuk, A. N. and J. E. Nelson. 2003. Developmental differences are correlated with relative brain size in birds: a comparative analysis. Can. J. Zoolog. 81:1913-1928.

519 Jetz, W., G. Thomas, J. Joy, K. Hartmann and A. Mooers. 2012. The global diversity of birds in space and time. Nature 491:444-448.

521 Lashley, K. S. 1949. Persistent problems in the evolution of mind. Q. Rev. Biol. 24:28-42.

522 Lefebvre, L., S. M. Reader and D. Sol. 2004. Brains, innovations and evolution in birds and primates. $523 \quad$ Brain Behav. Evolut. 63:233-246.

524 Lefebvre, L. and D. Sol. 2008. Brains, lifestyles and cognition: are there general trends? Brain Behav. 525 Evolut. 72:135-144.

526 Lefebvre, L. 2013. Brains, innovations, tools and cultural transmission in birds, non-human primates, and fossil hominins. Front. Hum. Neurosci. 7:10-3389.

Mazerolle, M. J. 2015. AICcmodavg: Model selection and multimodel inference based on (Q)AIC(c). R package version 2.0-3. http://CRAN.R-project.org/package=AICcmodavg.

McGuire, L. P. and J. M. Ratcliffe. 2011. Light enough to travel: migratory bats have smaller brains, but not larger hippocampi, than sedentary species. Biol. Lett. 7:233-236.

Mitchell, T. D. and P. D. Jones. 2005. An improved method of constructing a database of monthly climate observations and associated high-resolution grids. Int. J. Climatol., 25:693-712.

Newton, I. 2008. The ecology of bird migration. Academic Press, London, U.K.

Orme, D., Freckleton, R., Thomas, G., Petzoldt, T., Fritz, S., Isaac, N., and Pearse, W. 2014. caper: Comparative Analyses of Phylogenetics and Evolution in R, R package version 0.5.2; 2013. http://CRAN.R-project.org/package=caper

Piersma, T. and A. Lindström. 1997. Rapid reversible changes in organ size as a component of adaptive behaviour. Trends Ecol. Evol. 12:134-138. 
540 Pravosudov, V. V. and N. S. Clayton. 2002. A test of the adaptive specialization hypothesis: population 541 differences in caching, memory, and the hippocampus in black-capped chickadees (Poecile atricapilla). Behav. Neurosci. 116:515.

543 Pravosudov, V. V., K. Sanford and T. P. Hahn. 2007. On the evolution of brain size in relation to 544 migratory behaviour in birds. Anim. Behav. 73:535-539.

545 R Core Team. 2015. R: A Language and Environment for Statistical Computing. R Foundation for 546 Statistical Computing, Vienna, Austria.

547 Reader, S. M. and K. N. Laland. 2002. Social intelligence, innovation, and enhanced brain size in 548 primates. Proc. Natl. Acad. Sci. USA 99:4436-4441. 405 .

Roth, T. C. and V. V. Pravosudov. 2009. Hippocampal volumes and neuron numbers increase along a gradient of environmental harshness: a large-scale comparison. Proc. R. Soc. Lond. B 276:401-

Roth, T. C., L. D. LaDage and V. V. Pravosudov. 2011. Variation in hippocampal morphology along an environmental gradient: controlling for the effects of day length. Proc. R. Soc. Lond. B rspb20102585.

Rothman, K. J. 1990. No adjustments are needed for multiple comparisons. Epidemiology. 1:43-46.

Rothman, K. J. 2014 Six persistent research misconceptions. J Gen Int Med. 29:1060-1064.

Rubolini, D., A. Liker, L. Z. Garamszegi, A. P. Møller and N. Saino. 2015. Using the BirdTree. org website to obtain robust phylogenies for avian comparative studies: A primer. Current Zool. 61:959-965.

Sayol, F., L. Lefebvre and D. Sol. 2016. Relative Brain Size and Its Relation with the Associative Pallium in Birds. Brain Behav. Evolut. 87:69-77.

Schuck-Paim, C., W. J. Alonso and E. B. Ottoni. 2008. Cognition in an ever-changing world: Climatic variability is associated with brain size in neotropical parrots. Brain Behav. Evolut. 71:200215.

Sol, D., N. Garcia, A. Iwaniuk, K. Davis, A. Meade, W. A. Boyle and T. Székely. 2010. Evolutionary divergence in brain size between migratory and resident birds. PloS ONE 5:e9617.

Sol, D., L. Lefebvre and J. D. Rodríguez-Teijeiro. 2005. Brain size, innovative propensity and migratory behaviour in temperate Palaearctic birds. Proc. R. Soc. Lond. B 272:1433-1441.

Sol, D., T. Székely, A. Liker, L. Lefebvre. 2007. Big-brained birds survive better in nature. Proc. R. 
Soc. Lond. B 274:763-769.

571 Sol, D. 2009. Revisiting the cognitive buffer hypothesis for the evolution of large brains. Biol. Lett. 5:130-133.

573 Timmermans, S., L. Lefebvre, D. Boire and P. Basu. 2000. Relative size of the hyperstriatum ventrale is 574 the best predictor of feeding innovation rate in birds. Brain Behav. Evolut. 56:196-203.

575 Vágási, C. I., P. L. Pap, O. Vincze, G. Osváth, J. Erritzøe and A. P. Møller. 2016. Morphological 576 adaptations to migration in birds. Evol. Biol. 43: 48-59.

577 Vincze, O., I. C. Vágási, P. L. Pap, G. Osváth and A. P. Møller. 2015. Brain regions associated with $578 \quad$ visual cues are important for bird migration. Biol. Lett. 11:20150678.

579 Winkler, H., B. Leisler and G. Bernroider. 2004. Ecological constraints on the evolution of avian brains. $580 \quad$ J. Ornithol. 145:238-244. 
Table 1. Models exploring the relationship between brain size and migration distance in birds with different migratory strategies. Each row represents a separate model. All models include body mass as covariate (effect of body mass not shown).The first column provides the criteria used to define the species pool for each model. $\mathrm{t}$ - and P-values shown here are weighted averages over 100 PGLS models with different phylogenetic trees. The sign of $\mathrm{t}$ value indicates the direction of the association, while their value show the strength of association. Results from analyses of the entire taxonomic range and restricted analyses of passerines are provided.

\begin{tabular}{|c|c|c|c|c|c|c|c|c|}
\hline \multirow[b]{2}{*}{ Migration distance restriction } & \multicolumn{4}{|c|}{ Entire species pool } & \multicolumn{3}{|c|}{ Passerine species } & \multirow[b]{2}{*}{$\mathbf{R}^{2}$} \\
\hline & $\mathbf{n}$ & t-value & P-value & $\mathbf{R}^{2}$ & $\mathbf{n}$ & t-value & P-value & \\
\hline No restriction & 1466 & -5.37 & $<0.0001$ & 0.89 & 610 & -6.44 & $<0.0001$ & 0.90 \\
\hline$>0 \mathrm{~km}$ & 529 & -3.11 & 0.0022 & 0.90 & 189 & -4.22 & $<0.0001$ & 0.92 \\
\hline$>500 \mathrm{~km}$ & 387 & -3.60 & 0.0004 & 0.90 & 143 & -4.49 & $<0.0001$ & 0.92 \\
\hline$>1,000 \mathrm{~km}$ & 326 & -2.74 & 0.0067 & 0.92 & 119 & -3.82 & 0.0002 & 0.93 \\
\hline$>2,000 \mathrm{~km}$ & 233 & -3.60 & 0.0004 & 0.92 & 78 & -3.42 & 0.0010 & 0.92 \\
\hline$>0 \&<500 \mathrm{~km}$ & 142 & 2.21 & 0.0319 & 0.92 & 46 & 2.47 & 0.0179 & 0.95 \\
\hline$>500 \&<1,000 \mathrm{~km}$ & 61 & 0.78 & 0.4415 & 0.87 & 24 & 0.48 & 0.6434 & 0.93 \\
\hline$>2,000 \mathrm{~km} \&$ tropical wintering & 146 & -3.32 & 0.0012 & 0.92 & 58 & -3.34 & 0.0015 & 0.90 \\
\hline
\end{tabular}


Table 2. Models exploring the relationship between brain size and non-breeding minimum temperature, seasonality or non-breeding latitude in birds with different migratory strategies. Each row represents a separate model. All models include body mass as covariate (effect of body mass not shown). The first column provides the criteria used to define the species pool each model was based on. Where two sample sizes are given, the first refers to the minimum temperate and the seasonality models, while the second to the non-breeding latitude model. t- and P-values shown here are weighted averages over 100 PGLS models with different phylogenetic trees. The sign of $t$ value indicates the direction of the association, while their value show the strength of association. Results from analyses of the entire taxonomic range and restricted analyses of passerines are provided.

\begin{tabular}{|c|c|c|c|c|c|c|c|c|c|c|}
\hline \multirow{2}{*}{\begin{tabular}{|c|} 
\\
$\begin{array}{c}\text { Migration distance } \\
\text { restriction }\end{array}$ \\
\end{tabular}} & \multirow[b]{2}{*}{$\mathbf{n}$} & \multicolumn{3}{|c|}{$\begin{array}{c}\text { Non-breeding minimal } \\
\text { temperature }\end{array}$} & \multicolumn{3}{|c|}{ Seasonality } & \multicolumn{3}{|c|}{ Non-breeding latitude } \\
\hline & & t-value & P-value & $\mathbf{R}^{2}$ & t-value & P-value & $\mathbf{R}^{2}$ & t-value & P-value & $\mathbf{R}^{2}$ \\
\hline \multicolumn{11}{|l|}{ Entire species pool } \\
\hline $0 \mathrm{~km}$ & $934 / 937$ & -2.55 & 0.0134 & 0.89 & 0.53 & 0.6029 & 0.89 & 0.34 & 0.7274 & 0.89 \\
\hline$<500 \mathrm{~km}$ & $1076 / 1079$ & -3.50 & 0.0007 & 0.89 & 1.38 & 0.1794 & 0.89 & 1.27 & 0.2153 & 0.89 \\
\hline $0>\&<500 \mathrm{~km}$ & 142 & -4.14 & 0.0001 & 0.93 & 2.84 & 0.0060 & 0.93 & 3.84 & 0.0002 & 0.93 \\
\hline$>500 \&<1,000 \mathrm{~km}$ & 61 & -1.83 & 0.0725 & 0.88 & 1.99 & 0.0524 & 0.88 & 2.17 & 0.0344 & 0.88 \\
\hline \multicolumn{11}{|l|}{ Passerines } \\
\hline $0 \mathrm{~km}$ & 421 & -2.77 & 0.0062 & 0.90 & 0.84 & 0.4021 & 0.90 & 1.79 & 0.0752 & 0.90 \\
\hline $0>\&<500 \mathrm{~km}$ & 46 & -4.47 & 0.0001 & 0.97 & 3.36 & 0.0017 & 0.96 & 3.42 & 0.0014 & 0.96 \\
\hline$>500 \&<1,000 \mathrm{~km}$ & 24 & -1.07 & 0.2974 & 0.94 & 0.47 & 0.6453 & 0.94 & 1.36 & 0.1871 & 0.94 \\
\hline
\end{tabular}




\section{FIGURE LEGENDS}

599 Figure 1. Map illustrating geographic data coverage. Orange circles represent the geometric centroid of the breeding areas, green pluses 600 represent the geometric centroids of the wintering grounds of the studied species.

601

602 Figure 2. Association between migration distance and body-mass controlled residual brain weight in the entire species list (A), in species 603 with migration distance between 0 and 1,000 km (B), and in species with migration distance over 2,000 km (C). Figures D-E-F show the 604 same for passerines only. Note that figures are based on raw data points, therefore much of the variation can be accounted to phylogenetic 605 effects.

606

607 Figure 3. The association between non-breeding minimum temperature and body-mass controlled residual brain weight in fully resident 608 species (A), in species with migration distance between 0 and $500 \mathrm{~km}$ (B), and in species with migration distance between 500 and $1,000 \mathrm{~km}$ 609 (C). Figures D-E-F show the same for passerines only. Note that figures are based on raw data points, therefore much of the variation can be 610 accounted for by phylogenetic effects (e.g. green filled circles on figure 3A,B,C denote species from the Galliformes bird order). 